\title{
Presynaptic control of transmission through group II muscle afferents in the midlumbar and sacral segments of the spinal cord is independent of corticospinal control
}

\author{
N. C. Aggelopoulos $\cdot$ S. Chakrabarty $\cdot$ S. A. Edgley
}

Received: 12 March 2007 / Accepted: 10 January 2008 / Published online: 30 January 2008

(C) Springer-Verlag 2008

\begin{abstract}
Transmission of information from the terminals group II muscle afferents is subject to potent presynaptic modulation by both segmental group II and cutaneous afferents and by descending monoaminergic systems. Currently it is unknown whether descending corticospinal fibres affect this transmission. Here we have examined whether corticospinal tract activation modulates the size of monosynaptic focal synaptic potentials (FSPs) evoked by group II muscle afferents, and the excitability of intraspinal terminals of group II afferents, both of which are indices used to show presynaptic control. Conditioning stimulation of corticospinal pathways had no effects on the sizes of group II evoked FSPs in the midlumbar or sacral segments at either dorsal horn or intermediate zone locations. These stimuli also had no effect on the excitability of single group II afferent terminals in the dorsal horn of the midlumbar segments. As positive controls, we verified that the corticospinal conditioning
\end{abstract}

N.C. Aggelopoulos and S. Chakrabarty equally contributed to this work.

N. C. Aggelopoulos · S. Chakrabarty · S. A. Edgley Department of Anatomy, University of Cambridge,

Downing Street, Cambridge CB2 3DY, UK

N. C. Aggelopoulos

Max Planck Institute for Biological Cybernetics,

Tuebingen, Germany

S. Chakrabarty

Centre for Neurobiology and Behaviour,

Columbia University, New York, USA

S. A. Edgley $(\square)$

Department of Physiology Development and Neuroscience,

University of Cambridge, Downing Street,

Cambridge CB2 3DY, UK

e-mail: sae1000@cam.ac.uk stimuli used did effectively depress FSPs evoked from cutaneous afferents recorded at the same spinal locations as the group II field potentials in all experiments. Corticospinal tract conditioning stimuli did not consistently enhance or reduce the depression of group II FSPs that was evoked by stimulation of ipsilateral segmental group II or cutaneous afferents; in the large majority of cases there was no effect. The results reveal that the control of transmission of information from group II afferents in these regions of the spinal cord is independent of direct corticospinal control.

\section{Introduction}

As a general principle the large majority of somatosensory primary afferents are subject to presynaptic modulation of transmission (see Rudomin and Schmidt 1999 for review). The systems that produce this inhibition have specific input patterns in different types of afferent and at different locations in the spinal cord. In addition, different spinal segments have specialised organisation and functions. One example of this is the projection of information from group II muscle afferents to the spinal cord. In the main segments of the lumbar enlargement (L6, L7 and S1) group II afferents from the major groups of limb muscles evoke relatively small focal synaptic potentials (FSPs, see Fu et al. 1974). In contrast, much more prominent FSPs are evoked by group II afferents of specific nerves in two specific regions: the dorsal horn and intermediate zone of the midlumbar segments (L4 \& rostral L5; see Edgley and Jankowska 1987a), and the dorsal horn of the lower sacral segments (S1-S2; see Jankowska and Riddell 1993). At both of these locations large synaptic actions are evoked by group II muscle afferents, and many neurones are activated by these stimuli. These synaptic actions are under a powerful 
presynaptic control, which has been studied with a range of techniques including dorsal root potential recordings, intraspinal threshold changes at single afferent terminals, intraaxonal recording from single afferent axons, or as a depression of monosynaptic FSPs (Harrison and Jankowska 1989; Riddell et al. 1995; Bras et al. 1990; Jankowska and Riddell 1995, 1998; Jankowska et al. 2000, 2003). Major sources of presynaptic inhibition in group II afferents at these locations are group II muscle afferents themselves and cutaneous afferents. Stimulation of group I muscle afferents however, has no effect.

Well-studied and distinct systems mediate presynaptic inhibition of the terminals of muscle spindle primary (group Ia) afferents and Golgi tendon organ (group Ib) afferents (see Rudomin and Schmidt 1999 for review). Both Ia and Ib afferents contribute to their own presynaptic depression, but presynaptic inhibition is evoked at terminals of $\mathrm{Ib}$ afferents by cutaneous afferents, whereas the same stimuli release most group Ia afferents from presynaptic inhibition (i.e. the systems producing presynaptic inhibition of Ia afferents are inhibited, for review see Rudomin and Schmidt 1999). Furthermore, the corticospinal system usually evokes presynaptic inhibition on Ib, but not Ia afferents (see Rudomin et al. 1986; Enríquez et al. 1996; Eguibar et al. 1997). In an early study in the lower lumbar segments (L6-S1, Carpenter et al. 1963) primary afferent depolarisation (PAD) was evoked by trains of electrical stimuli delivered in the hindlimb area of the sensorimotor cortex in half $(6 / 12)$ of the group II afferent terminals tested. This suggests that the segmental system exerting presynaptic control over group II afferents resembles the system exerting presynaptic control over $\mathrm{Ib}$ afferents (i.e. both cutaneous and corticospinal systems evoke presynaptic inhibition in the terminals).

In this study we have investigated the effects of corticospinal tract stimulation on the transmission of information from group II afferent terminals in the midlumbar and sacral segments, where they evoke large synaptic field potentials, reflecting actions on specific groups of neurones, and at both dorsal horn and intermediate zone locations (Edgley and Jankowska 1987a, b). Our results indicate that transmission from group II afferents at these locations is not influenced by specific stimulation of corticospinal fibres, thus differing from the general pattern shown by Ib afferents. Some of these results have been described in an abstract (Aggelopoulos et al. 2000).

\section{Methods}

The observations reported here are drawn from experiments performed under general anaesthesia on eleven adult male cats $(4.25-5.25 \mathrm{~kg})$. All experiments were done under UK
Home Office regulations (Animals Scientific Procedures act 1986), and were approved by the local ethical committee. Anaesthesia was induced with a mixture of ketamine and xylazine $\left(15 \mathrm{mg} \mathrm{kg}^{-1}\right.$ and $1 \mathrm{mg} \mathrm{kg}^{-1}$, respectively, delivered i.m.), and subsequently maintained with pentobarbitone (2.4-4.3 $\mathrm{mg} \mathrm{kg}^{-1} \mathrm{hr}^{-1}$ i.v.). Blood pressure and heart rate were monitored via a cannula inserted into the right femoral artery and remained between $80-150 \mathrm{~mm} \mathrm{Hg}$ at all times. Body core temperature was maintained at $37-38^{\circ} \mathrm{C}$ with a homeothermic blanket and a servo-controlled radiant heating system. A tracheal cannula was inserted following a tracheotomy. Selected nerves in the left hindlimb were dissected free to allow electrical activation of peripheral afferents. These always included the quadriceps (Q) and sartorius (Sart.), tibialis anterior (TA), extensor digitorum longus (EDL) gastrocnemius-soleus (GS), posterior bicepssemitendinosus (PBST). The cutaneous sural (Sur.), superficial peroneal (SP) and Saphenous nerves were also taken. To prevent drying a pool was made from skin flaps and was filled with warm mineral oil. A laminectomy was performed to expose the dorsal surface of the spinal cord from the L3 vertebra to the sacrum. In five experiments paralysis was induced with pancuronium (initial dose of $0.5-0.7 \mathrm{mg}$ $\mathrm{kg}^{-1}$ i.v., supplemented with additional doses of 0.25 $0.35 \mathrm{mg} \mathrm{kg}^{-1}$ ) and artificial ventilation was applied. Adequacy of anaesthesia during paralysis was assessed by checking regularly that blood pressure and heart rate were unaltered by noxious stimuli, and also that the pupils were constricted and remained so after these stimuli. The other six animals were not paralysed during recording, but were artificially ventilated.

Focal synaptic potentials (FSPs)

The synaptic actions evoked by afferent fibres were recorded as extracellular focal synaptic potentials (FSPs). These were evoked by stimulation of peripheral nerves (square pulses, $0.2 \mathrm{~ms}$ duration, $0.5 \mathrm{~Hz}$ repetition rate) at strengths expressed as multiples of the threshold of the most excitable fibres in the nerve $(\mathrm{T})$. In muscle nerves stimuli at strengths up to $1.5 \mathrm{~T}$ activate only group I fibres, group II afferents begin to be recruited at strengths of 1.6$1.8 \mathrm{~T}$, up to $5 \mathrm{~T}$ (Matthews 1972; Jack 1978; Lundberg et al. 1986). Cord dorsum potentials (CDPs) recorded with silver ball electrodes were used to set the thresholds for nerve stimulation and to monitor the conduction in segmental and descending cortical afferents. Previous studies have described the characteristic FSPs evoked by group II afferents at different levels of the lumbosacral cord (Edgley and Jankowska 1987a; Jankowska and Riddell 1993; Riddell and Hadian 2000a). These were recorded via glass microelectrodes filled with $2 \mathrm{M} \mathrm{NaCl}$, impedance $0.5-4 \mathrm{M} \Omega$. The effects of conditioning stimulation were examined on the 
amplitude of the initial rising phase of the FSPs, which represents the monosynaptic component.

Corticospinal fibres were stimulated through monopolar tungsten or steel electrodes (125 $\mu \mathrm{m}$ diameter, varnish insulated, tips exposed to an impedance of 50-200 k $\Omega$ ) inserted into the contralateral pyramidal tract (PT) in the low medulla. Electrodes were positioned in the PT, entering the medulla $0.5 \mathrm{~mm}$ lateral and $1-2 \mathrm{~mm}$ rostral to the obex, with an angle of $30^{\circ}$, tip rostral. Activation of corticospinal fibres was assessed by monitoring both antidromic potentials recorded with silver ball electrodes from the surface of the sensorimotor cortex and from the growth of the dispersed orthodromic volleys recorded from the lateral surface of the low thoracic spinal cord (which required averaging). We took particular care to ensure that the stimuli we used did not activate reticulospinal axons. As the electrode was lowered through the medulla, stimulation could evoke large descending reticulospinal volleys from the region of the MLF and gigantocellular reticular formation (see Edgley et al. 2004). As the electrode approached the PT these large descending volleys declined and disappeared 1-2 $\mathrm{mm}$ above the best point for activating corticospinal fibres. We ensured that at the final location of the PT electrode there were no visible descending reticulospinal volleys at the maximal stimulus intensities used.

Recordings were made at sites where large FSPs could be evoked, but where there were no large single unit responses. Control unconditioned FSPs were averaged and compared to FSPs preceded by conditioning stimuli (Riddell et al. 1995; Jankowska and Riddell 1998). The conditioning stimuli were trains of 3-5 stimuli to the PT $(0.2 \mathrm{~ms}$ pulses, $2.5-3.5 \mathrm{~ms}$ intervals), delivered $30-50 \mathrm{~ms}$ before the test stimulus. The sizes of monosynaptic FSPs were assessed as the amplitude of the initial rising phase of the potential (0.2-1.0 ms from onset) from overlaid averages of 20 sweeps. Conditioned FSPs were compared with multiple control averages, used to ensure stability of the control FSP and that the effect was consistently evoked.

In addition to the effects of PT stimulation on the FSPs, we also examined the effects of these stimuli on the reduction of FSPs evoked by conditioning stimulation of segmental cutaneous and group II afferents by spatial facilitation, in which case the preconditioning PT stimuli were delivered between 0 and $20 \mathrm{~ms}$ before the conditioning stimuli, which themselves came $30-50 \mathrm{~ms}$ before the stimulus that evoked the FSP. As a criterion level, consistent depression of FSP amplitude by greater than $10 \%$ on repeated trials was accepted as representing a depression. Paired $t$-tests were carried out to determine whether there were significant changes to the conditioned responses. Multiple sets of interleaved conditioned and unconditioned responses were always recorded to ensure consistency.
Excitability testing procedure

In three experiments, filaments from which discriminable single unit action potentials from group II afferents could be recorded were separated from one of the branches of the deep peroneal nerve, usually TA, to determine whether PT stimulation could alter the threshold for activation of the intraspinal terminals. Axons in the nerve filament were antidromically activated by intraspinal stimuli and identified as group II afferents by collision from stimulation of the common peroneal nerve at group II stimulus strength $(2-5 \mathrm{~T})$. The ventral roots were intact in these experiments but the stimuli used in the midlumbar segments (up to $33 \mu \mathrm{A}$ ) were too weak to have activated motoneurones in the branches of the deep peroneal nerve, which are located in the caudal L6 and L7 segments. For units with antidromic thresholds of less than $10 \mu \mathrm{A}$, a threshold hunting circuit was used to follow the excitability of the intraspinal terminal (see Riddell, Jankowska and Eide 1993). The threshold hunting circuit we used had a limited current passing ability (less than $10 \mu \mathrm{A})$ so for afferents with intraspinal thresholds near or above this we used systematic spike counting to assess the probability that a given stimulus would evoke a spike (firing index). For these units (thresholds of 5-33 $\mu \mathrm{A}$ ) a fixed, just subthreshold, stimulus intensity was used, where responses were evoked in $2 / 10$ stimuli or fewer. Changes in response probability (firing index) evoked by conditioning stimuli were measured. The initial stimulus intensity was chosen so that conditioning stimulation of group II afferents ( $Q$ or $S$ ) increased the firing index. Blocks of 10 or 20 stimuli with and without conditioning were assessed; these were always repeated twice to ensure stability. Conditioning stimuli were delivered to the PT (range of intervals $30-50 \mathrm{~ms}$ prior to a test stimulus) as described above for the FSPs. Changes in terminal excitability were also evoked from segmental cutaneous and muscle afferents, and the interaction of PT and segmental inputs was also examined by delivering preconditioning stimuli to the PT at intervals prior to conditioning stimuli to the segmental afferents.

\section{Histological examination}

At the end of each experiment, electrolytic lesions were made to mark the PT electrode tips, animals were killed by overdose of anaesthetic and perfused with $4 \%$ paraformaldehyde. Counterstained $100 \mu \mathrm{m}$ thick histological sections were cut from the spinal cord and the lower medulla. These were used to verify the locations of the recording sites in the spinal cord and stimulation sites in the pyramidal tract from the electrode tracks. 


\section{Results}

Focal synaptic potentials from the midlumbar segments

Recordings were made at several different segmental levels in each experiment. Recordings were made both from dorsal horn sites where group II muscle afferents and cutaneous afferents evoked large, short latency FSPs, and from intermediate zone sites where FSPs evoked by group I afferents usually accompany longer latency FSPs evoked by group II afferents (Edgley and Jankowska 1987a, b; Jankowska and Riddell 1993; Riddell and Hadian 2000a). In total FSPs were recorded from 14 dorsal horn sites and 14 intermediate zone sites in the midlumbar segments (L4 segment and the rostral third of the L5 segment). At most sites FSPs were evoked from more than one muscle nerve, making a total of 31 dorsal horn FSPs and 24 intermediate zone FSPs.

At dorsal horn locations, large FSPs were usually evoked by stimulation of Q, Sart., and TA at 2-5 T, as described previously (Edgley and Jankowska 1987a). These group II FSPs could be substantially depressed by conditioning stimulation of $\mathrm{Q}$, Sart. and TA group II muscle afferents (e.g. Fig. 1a), but not by stimuli at strengths that activated only group I muscle afferents $(<1.5 \mathrm{~T})$, as reported previously by Riddell et al. (1995). Monosynaptic group II FSPs at many locations in this region were also depressed by a single stimulus to cutaneous afferents (Sur 10/13 FSPs, 77\%; SP 26/30 FSPs, 87\%). In contrast, stimulation of the contralateral PT with trains of 3-5 stimuli at strengths that produced maximal antidromic potentials in the motor cortex did not affect the monosynaptic group II muscle afferent FSPs at any of the sites studied. This is illustrated in Fig. 1b, where the control and conditioned FSPs are superimposed. For comparison the reduction of the same FSP by conditioning stimulation to Q group II afferents is shown in Fig. 1a. This was a consistent finding for all 31 FSPs tested.

At most intermediate zone locations in the L4 and rostral L5 segments both early group I and later group II FSPs were elicited by stimulation of muscle afferents (Fig. 1c-e), as described previously (Edgley and Jankowska 1987a). In agreement with previous observations (Riddell et al. 1995), midlumbar group I FSPs were sometimes depressed by stimulation of muscle afferents at strengths that activate group I muscle afferents (1.8-2 T) but were not further depressed by stimulation at higher intensities that recruited both group I and group II muscle afferents $(5 \mathrm{~T})$. The group I components of the FSPs in Fig. 1c, d show this depression. In contrast, group II FSPs were substantially reduced (Fig. 1c) and sometimes eliminated by conditioning stimuli that activated other group II afferents. At all sites sampled conditioning stimulation of at least one source of group II
A

DORSAL HORN

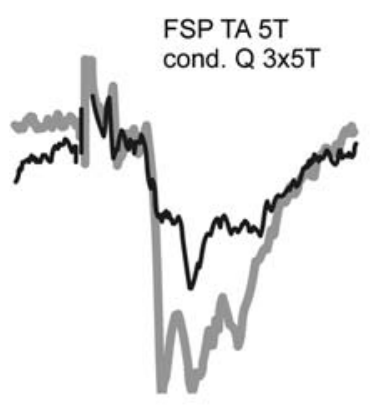

C INTERMEDIATE ZONE
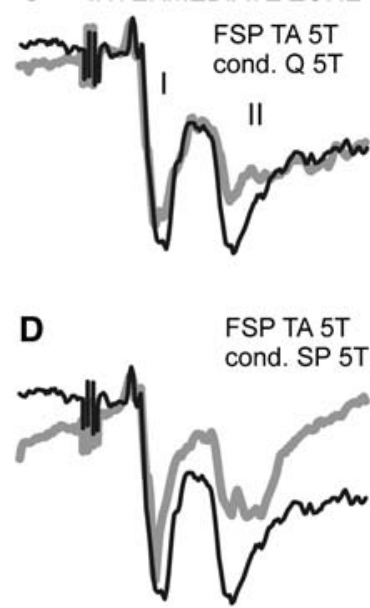

B

FSP TA 5 T

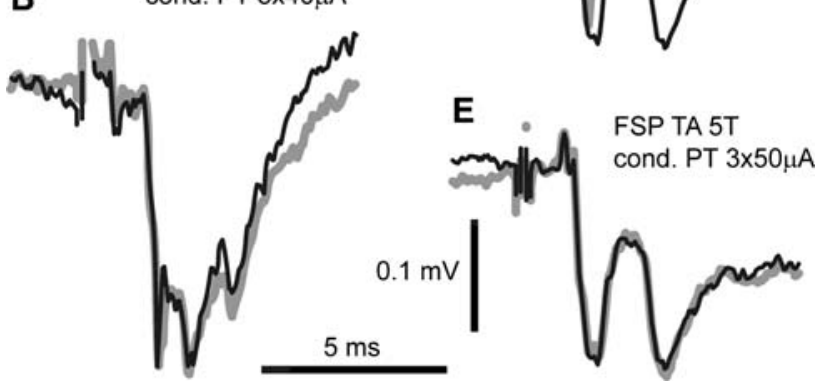

Fig. 1 Group II evoked FSPs in the L4 and rostral L5 segment were not depressed by conditioning stimulation of PT fibres. $\mathbf{a}$ and $\mathbf{b}$ show FSPs recorded from a dorsal horn location which were evoked by stimulation of the TA nerve at $5 \mathrm{~T}$. The monosynaptic group II FSP elicited by a test stimulus (heavy grey line) was reduced by conditioning stimulation to Q muscle afferents (a, thin black line), but not by PT stimulation (b, thin blackline). $\mathbf{c}-\mathbf{e}$ show an FSP evoked from TA group II afferents recorded from an intermediate zone site (thick grey lines), which has an early group I evoked component and a later group II evoked component, as indicated in c. The group II component is reduced by conditioning stimulation of group II afferents and by cutaneous afferents (c and $\mathbf{d}$, thin black line), but not by PT stimuli (e, thin black line). Field potentials evoked by the conditioning stimuli have not been removed from these traces

afferents evoked a statistically significant reduction in FSP amplitude and often all tested group II inputs produced suppression. Intermediate zone FSPs were also frequently depressed by activation of cutaneous afferents (from Sur 4/ 5 FSPs, 80\%; from SP 8/10 FSPs, 80\%), as illustrated in Fig. 1d. As in the dorsal horn, PT stimulation failed to produce a depression of any of the 24 monosynaptic group II afferent evoked FSPs tested in the L4 and rostral L5 segments in the intermediate zone. An example is shown in Fig. 1e.

\section{Positive control experiments}

The absence of effects of conditioning PT stimulation on group II FSPs could reflect a lack of corticospinal influence on the spinal circuitry responsible for the presynaptic depression of group II FSPs, but could also reflect inadequate or failed 
activation of the corticospinal axons. Evidence that PT stimulation effectively activated corticospinal axons came from the presence of descending volleys in the dorsolateral funiculi and antidromic field potentials in the motor cortex, and the fact that the PT stimuli evoked FSPs in the spinal grey matter suggesting that effective synaptic actions were evoked from their terminals (see e.g. Fig. 2a-d, top traces). As an important positive control, we therefore sought evidence that the PT stimuli that we used were able to influence

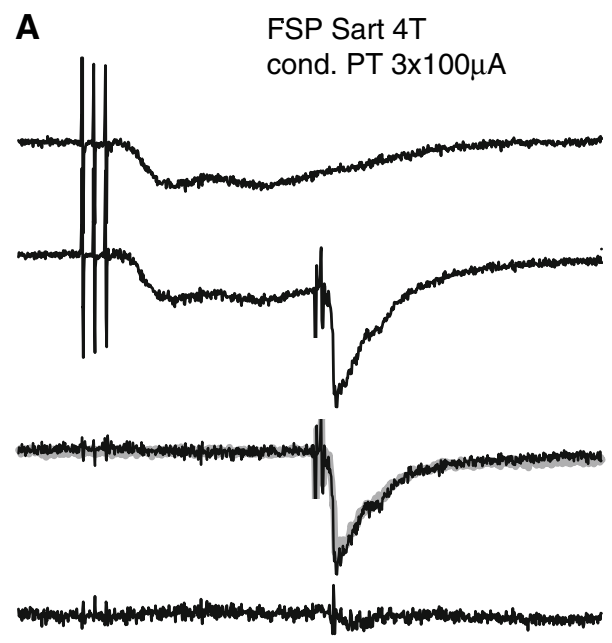

C

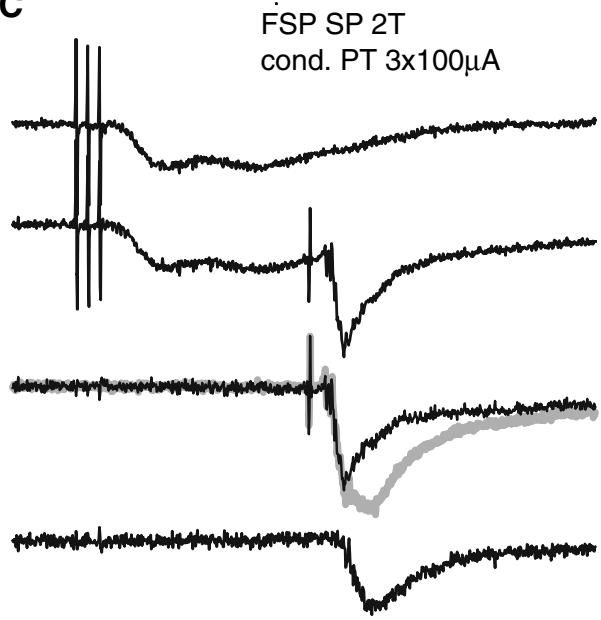

$0.2 \mathrm{mV} A \& \mathrm{C}$

Fig. 2 PT stimuli reduce cutaneous, but not group II evoked FSPs in the midlumbar segments. Examples of FSPs recorded from two different sites in two different experiments (a and $\mathbf{c} ; \mathbf{b}$ and $\mathbf{d}$ ) where maximal PT stimulation failed to depress the group II FSPs in the dorsal horn. a and $\mathbf{b}$ show FSPs evoked by stimulation of group II afferents. The top traces are extracellular field potentials evoked by PT stimulation. The second traces show the field potentials due to both test (group II) and conditioning (PT) stimuli. The third traces show the conditioned FSPs with the extracellular field potentials from the conditioning stimulation subtracted (thin black lines). These are superimposed onto the uncon- spinal presynaptic inhibition, by showing that they could suppress FSPs at the spinal cord at the locations where group II field potentials were tested. This came from observations on FSPs evoked by stimulation of cutaneous afferents, on which presynaptic actions of cortical stimulation are well established (Carpenter et al. 1963; Andersen et al. 1964). Cutaneous evoked FSPs were very effectively reduced by PT stimulation in every experiment, including cutaneous evoked FSPs recorded in the L4 and rostral L5 segments at

B

B $\quad$ FSP TA 4T cond. PT $3 \times 75 \mu \mathrm{A}$
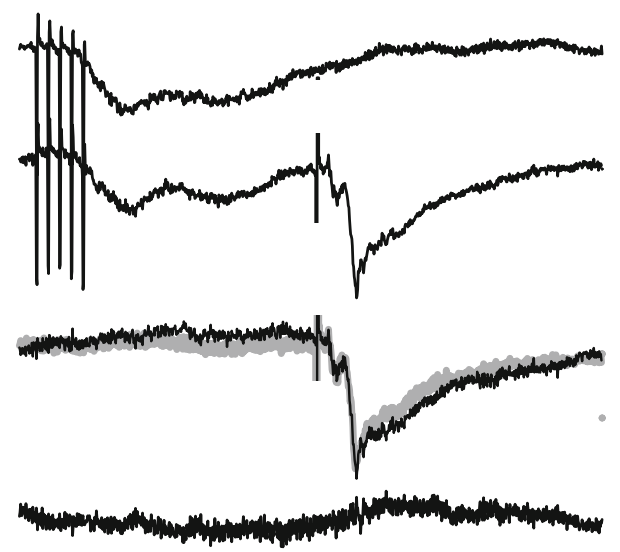

D

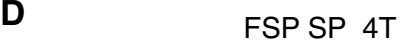
cond. PT $3 \times 75 \mu \mathrm{A}$

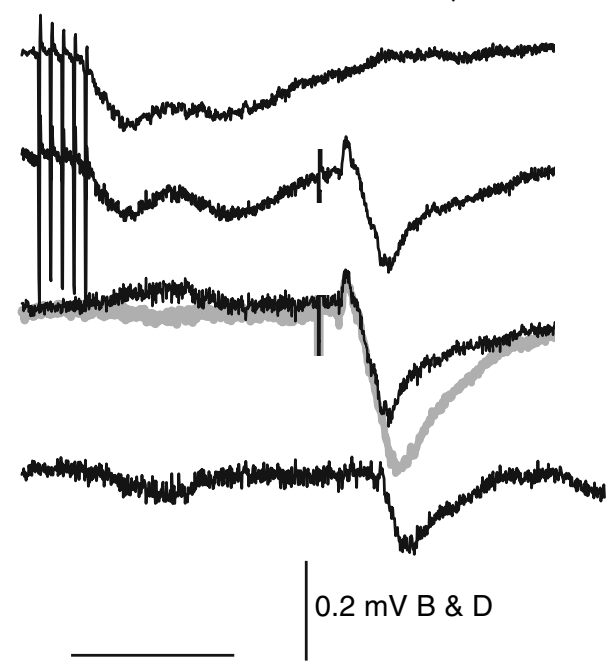

$10 \mathrm{~ms}$

ditioned test responses (thick grey lines). Finally, the bottom trace shows the difference of the two latter traces; the amount by which the unconditioned FSP exceeded the conditioned FSP. In both cases the lower line is flat indicating no depression of the FSP. In contrast, $\mathbf{c}$ and d show FSPs evoked by cutaneous afferents at the same sites as a and b. The sequence of traces is the same as in $\mathbf{a}$ and $\mathbf{b}$. In this case the superimposed conditioned and unconditioned traces are clearly different, as shown in the bottom difference traces. This positive control shows that the PT stimuli we used were able to reduce the FSPs evoked by cutaneous afferents 
the same sites at which group II evoked FSPs were unaffected by the same PT stimuli. Examples are shown in Fig. 2, where FSPs evoked from group II afferents (Fig. 2a, b) are unaffected by PT stimuli that substantially reduce the FSPs evoked from cutaneous afferents at the same sites (Fig. 2c, d). In all, ten FSPs evoked from the SP nerve and five from the Sur. nerve were tested in the L4 and rostral L5 segments. These were reduced by an average of $25 \pm 7 \%$ and $45 \pm 15 \%$ (mean $\pm \mathrm{SE}$ ) respectively. A further 18 cutaneous FSPs were tested in more caudal segments in the same experiments and similar reductions were consistently observed in every experiment. Thus the PT stimuli we used effectively activated spinal systems that were able to suppress cutaneous afferent evoked FSPs.

\section{Effects of PT stimulation on suppression of FSPs by segmental afferents}

Given the lack of effect of PT stimulation on group II FSPs, we examined whether stimulation of the PT could enhance or reduce the effects of conditioning stimulation of segmental afferents. At seven dorsal horn and six intermediate zone sites (27 FSPs) we delivered PT stimuli during trials in which a group II FSP was conditioned with a stimulus to other segmental group II afferents. This was to examine whether PT stimulation, although by itself ineffective, may have facilitatory or occlusive effects on the pathways mediating segmental presynaptic actions. The large majority of FSPs were unaffected by preconditioning with PT stimuli (Fig. 3): of 27 FSPs where conditioning group II stimuli produced a substantial reduction of FSP amplitude, additional conditioning stimuli delivered to the PT had no effect at $18(67 \%)$. The suppression was greater in four cases and smaller in five cases. The actions of these preconditioning stimuli to the PT were also examined on the reduction of FSPs evoked by conditioning stimulation of cutaneous afferents. There was no change in the size of the group II FSP in 10/16 FSPs tested (62.5\%). In four cases the suppression was greater and in two it was reduced. These data do not reveal a consistent effect of corticospinal fibres on the segmental pathways that suppress the FSPs, and in the majority of cases show no effect (Fig. 3b, e). In the population, the amplitude of the group II muscle afferent FSPs when conditioned by cutaneous nerve stimuli was not significantly altered by preconditioning PT stimuli (one-way analysis of variance, NS); this result was similar in both dorsal horn and intermediate zone locations for all nerves tested (SP and Sur, 13 dorsal horn FSPs and three intermediate zone FSPs, respectively).

\section{Measures of intraspinal excitability of group II afferents}

In addition to the FSP method for assessing presynaptic inhibition, the effects of PT stimulation were also examined
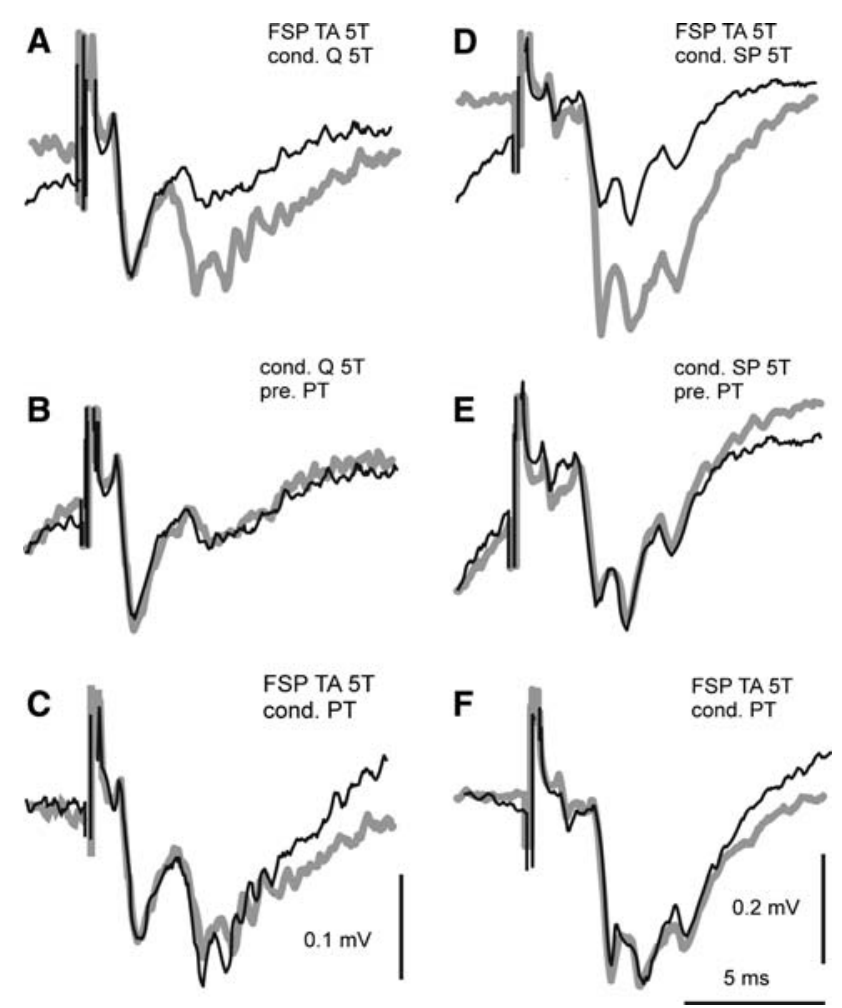

Fig. 3 Effects of preconditioning stimulation to the PT $(3 \times 100 \mu \mathrm{A})$ on the reduction of FSPs by segmental afferents. a-c show an FSP evoked from TA group II afferents recorded from the intermediate zone, comprising an initial group I evoked potential and a later group II potential. a shows that the unconditioned group II FSP (thick grey trace) is substantially reduced by conditioning stimulation of Q group II afferents (thin black trace). b shows that this conditioned FSP (grey trace) is not changed by additional preconditioning stimulation of the PT (thin black trace). $\mathbf{c}$ shows that the unconditioned FSP (grey trace) is unaffected by conditioning stimulation of the PT (thin black trace). Note that the group I FSP is unaltered by these stimuli. $\mathbf{d}-\mathbf{f}$ show group I and II FSPs evoked by stimulation of TA group II afferents at a dorsal horn location. d shows that the TA group II FSP (thick grey line) was depressed by conditioning stimulation of the SP nerve (thin black trace). e shows that this conditioned FSP (thick grey trace) is not further changed by preconditioning stimulation to the PT (thick black trace). f shows that the unconditioned FSP (thick grey trace) is not changed by conditioning stimulation to the PT (thin black trace)

on the thresholds for activation of group II afferents by intraspinal stimulation. For this we used 27 single group II axons dissected from the deep peroneal nerve which were antidromically activated by stimuli delivered in the L4 and rostral L5 segments. All were identified as group II afferents by collision with orthodromic volleys in the common peroneal nerve at stimulus intensities of between 1.7 and $5 \mathrm{~T}$ (mean $2.66 \pm 0.2 \mathrm{~T}$ ). The best location for eliciting antidromic action potentials was in the intermediate zone for six of these units (mean depth $2.3 \mathrm{~mm}$ from the surface), in the dorsal horn for the remaining 22 axons (mean depth $1.42 \mathrm{~mm}$ ). Changes in the firing index with a fixed stimulus intensity were used to determine terminal excitability in 16 of these units while 11 were tested with a 
threshold-hunting device (for details see Riddell et al. 1993).

The examples shown in Fig. 4 illustrate intraspinal stimulus intensities derived from a threshold hunting device. The activation thresholds of most of the single axons were decreased by single conditioning stimuli to segmental
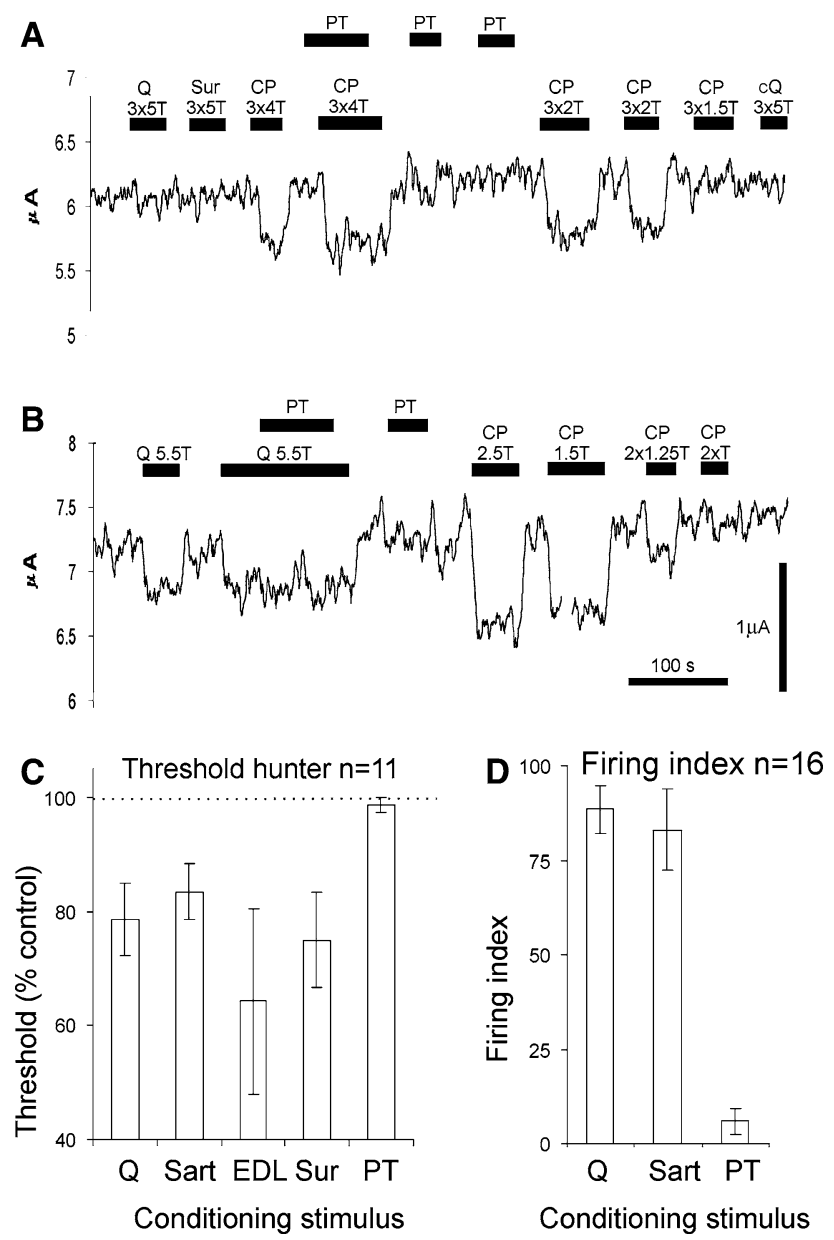

Fig. 4 Changes in the threshold for activation of TA group II muscle afferent terminals by intraspinal stimuli. $\mathbf{a}$ and $\mathbf{b}$ traces showing the changes in threshold for antidromic activation of two different group II muscle afferent terminals evoked by various stimuli. The threshold was tracked by a threshold hunter (see text) In a antidromic threshold was reduced by conditioning stimulation of Q group II afferents and by afferents from the common peroneal nerve, but not by the PT. Conditioning stimulation of the PT $(3 \times 100 \mu \mathrm{A})$ did not by itself lower the threshold nor did it alter the action of Q. In $\mathbf{b}$, stimuli to the common peroneal nerve produced a reliable reduction in the threshold for antidromic activation of this unit, but only with stimuli above $1.5 \mathrm{~T}$. Conditioning stimulation of the PT did not alter the threshold antidromic activation, or the reduction evoked from the $\mathrm{CP}$ nerve. $\mathbf{c}$ grouped data for 11 units tested with a threshold hunter. Changes in threshold are expressed relative to the control current $(100 \%)$. Stimulation of group II or cutaneous afferents reliably reduced the threshold, while stimulation of the PT did not. d intraspinal threshold tested with the firing index method for a group of 16 units. Stimulus intensity was set just subthreshold and changes in firing index measured when conditioning stimuli were applied (see text for further details). Stimulation of group II afferents from Q or Sart increased firing index, stimulation of the PT did not group II afferents (18/21 tested with Q, 12/14 tested with Sart.). In most of the afferents tested the activation threshold was also decreased by conditioning stimulation of cutaneous afferents (9/11 tested with SP, 3/6 tested with Sur). Figure $4 c, d$ quantify these effects for the fibres tested with a threshold hunter and by measuring firing index respectively. Where a threshold hunter was used, the intraspinal threshold (control range 2.5-9.5 $\mu \mathrm{A}$, mean $5.9 \pm 0.7 \mu \mathrm{A}$ ) was decreased by $20-30 \%$ by conditioning stimulation of group II or cutaneous afferents, but was not altered by PT stimulation (Fig. 4c). Where firing index was assessed (control intraspinal threshold 5-33 $\mu \mathrm{A}$, mean $16.8 \pm 0.2 .9 \mu \mathrm{A}$ ), the probability of evoking a spike for a given stimulus (firing index) was increased by stimulation of group II afferents, but was unaffected by PT stimulation (Fig. 4d). These observations are consistent with the lack of effect of conditioning stimuli to the PT on the group II evoked FSPs evoked in these segments. Also consistent with our observations on FSPs was the finding that conditioning stimulation to the PT did not detectably affect the changes in intraspinal threshold of group II afferent terminals that could be evoked by group II or cutaneous conditioning stimulation (Fig. 4a, b).

FSPs recorded in the sacral spinal cord S1, S2

Thirteen FSPs evoked from group II afferents were recorded at 9 different sites in the upper sacral segments of the spinal cord (S1 \& S2). These FSPs were evoked by group II afferents in the GS $(n=8)$ and PBST $(n=5)$ nerves. Monosynaptic group II GS FSPs were depressed by single conditioning stimuli to PBSt group II afferents (7/8 sites, $88 \%$ ) by a mean of $50 \pm 8 \%$ (mean \pm standard error) and by conditioning stimulation of Q group II afferents ( $8 / 8$ sites, $100 \%$ ) by a mean of $35 \pm 4 \%$ (Fig. $5 \mathrm{c}$ ). They were also depressed by single conditioning stimuli to cutaneous afferents; stimulation of Sur. at $2 \mathrm{~T}$ reduced the GS group II FSPs at $3 / 4$ sites $(75 \%)$ by $32 \pm 16 \%$. None of these FSPs was reduced by conditioning stimulation of the PT (Fig. 5a, b). As described above, positive controls of the effectiveness of the PT stimulation on cutaneous afferents was also examined at the sacral level (Fig. 5d). Such stimuli were able to depress FSPs evoked by SP and Sur. at the sacral level (5/12 tests, $42 \%)$.

\section{Discussion}

We have presented consistent evidence from 2 different approaches that monosynaptic FSPs evoked by group II afferent stimulation in the midlumbar segments (L4 and rostral L5) are not affected by conditioning stimulation of the PT. Additionally we show that FSPs evoked from group 

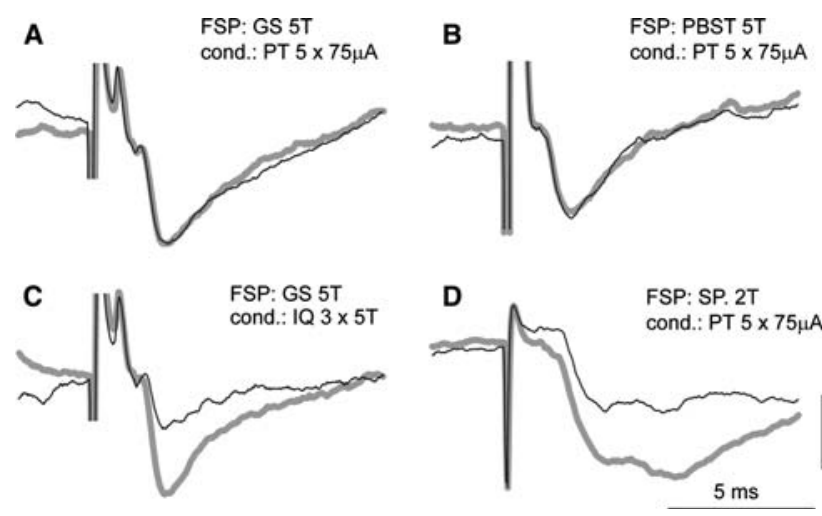

Fig. 5 FSPs evoked in the sacral segments. The records show FSPs evoked from a site in the caudal part of the S1 segment. Conditioned responses (thin black traces) are superimposed onto the unconditioned responses (thick grey traces). a and $\mathbf{b}$ show FSPs evoked by GS and PBST group II afferents recorded at this site that are unaffected by conditioning stimulation of the PT. $\mathbf{c}$ shows that conditioning stimulation of Q reduces the amplitude of the FSP evoked by stimulation of GS group II afferents. d shows that at the same site, cutaneous FSPs evoked from SP are substantially reduced by the same PT stimulation that was ineffective on group II evoked FSPs (in $\mathbf{a}$ and $\mathbf{b}$ )

II afferents of different nerves in more caudal (sacral) parts of the lumbosacral enlargement were also unaffected by stimulation of the PT.

The rationale behind these experiments follows previous work that has used reductions in FSPs to monitor presynaptic inhibition (Sypert et al. 1980; Riddell et al. 1995). Riddell et al. (1995) also observed the depression of monosynaptic group II FSPs in parallel with excitability testing of single axon terminals. Their conclusion was that monosynaptic FSPs represent synaptic currents, so the depression of these FSPs was an indirect but reliable means of assessing presynaptic inhibition of group II afferents evoked by segmental afferents. These arguments are based on the fact that the initial rising phase of the FSP is generated monosynaptically, so should represent synaptic current generated by group II afferents. This was supported by the observation that parallel effects were evoked by conditioning stimuli on the threshold for activation of group II axon terminals in the spinal cord and group II FSP amplitudes, by the latency of the effects after conditioning stimulation and from the parallel observations on intra- and extracellular records from single neurones (Riddell et al. 1995). In the current work we have used the same monosynaptic FSPs and measured the reduction of the initial components of them. The lack of effect of PT stimulation on FSPs in the rostral (L4 and rostral L5) and caudal (sacral) parts of the grey matter of the lumbosacral enlargement imply that the mechanisms producing presynaptic inhibition on group II afferent terminals are not influenced by fibres of the PT. Our positive control evidence is important and shows that this was a genuine absence of an action from the PT termi- nals. The PT stimuli did evoke synaptic actions in the lumbosacral cord, since they evoked local FSPs in the grey matter. Most importantly, the PT stimuli effectively depressed FSPs evoked by stimulation of cutaneous afferents at all segmental levels, including the same sites where group II FSPs were evoked. The absence of effects in L4rostral L5 and in the sacral segments is thus a genuine absence of a corticospinal effect, rather than an experimental failure to activate the descending fibres. For single group II afferent fibres terminating in the L4 segments this conclusion is also supported by the observation that the threshold for antidromic activation by intraspinal stimuli was unaffected by conditioning stimulation to the PT, but could be reduced by stimulation of group II afferents.

The interneuronal systems that control sensory transmission from group II afferents at different levels in the lumbosacral cord have some common features, in that group II FSPs at all levels are reduced by stimulation of group II and cutaneous afferents. In the L4 and rostral L5 segments and also in the sacral segments FSPs evoked from group II afferents were unaffected by PT stimulation. Previous findings (Carpenter et al. 1963) indicate that stimulation of the sensorimotor cortex was able to increase the excitability of group II muscle afferents from GS in the lower lumbar segments, although the terminal excitability of half of the group II afferent terminals tested was unaffected. Two possible explanations for the difference between our findings and those of Carpenter et al. (1963) are that there is a real difference between the group II terminals tested (from different nerves and at different spinal levels), or that the stimuli used by Carpenter et al. (1963) produced effects which were not mediated through the corticospinal tract, but by other descending pathways (e.g. cortico-bulbospinal). We attempted to examine whether this was the case in these experiments by stimulating the sensorimotor cortex and internal capsule directly, but were unable to produce a suppression of the monosynaptic group II evoked FSP's we tested. This limited set of observations suggests that the indirect pathways do not reduce FSPs significantly more than direct corticospinal pathways, in our experimental situation. Another possibility is that there is a real segmental difference between the control of group II afferent terminals; there appear to be very different circuitries at different spinal segmental levels and there are other indications that presynaptic inhibitory circuits are locally specific (Lomeli et al. 1998; Jankowska et al. 2000). In the midlumbar segments there are considerable differences between the presynaptic control of dorsal horn and intermediate zone and ventral horn terminals (Jankowska et al. 2002a, b; Edgley et al. 2003). There also seem to be different functional properties of circuits at these different levels, for example, the crossed inhibition evoked by group II muscle afferents in hindlimb motoneurones (Arya et al. 1991) is largely dependent on the 
midlumbar segments (Aggelopoulos and Edgley 1995), although there are neurones located more caudally with group II inputs (Riddell and Hadian 2000a, b).

A surprising finding was that PT stimuli did not affect the reduction of group II FSPs evoked by conditioning stimulation of either group II afferents or cutaneous nerve stimulation. This implies that not only do PT fibres not evoke presynaptic inhibition at group II afferent terminals, they do not powerfully influence the presynaptic inhibition evoked by segmental mechanisms at these terminals. Once again the positive control observations that the PT stimuli did effectively reduce cutaneous FSPs in all experiments shows that the absence of an effect was not due to inadequate PT activation. These results are surprising in that stimulation of cutaneous afferents could depress group II field potentials, and since PT stimulation depressed the cutaneous field potentials, it would be expected that the PT stimulation would reduce the depression of group II field potentials evoked by cutaneous afferents. A possible explanation for this lies in the heterogeneity of cutaneous afferents: it is possible that the type of cutaneous afferents that mediate the depression of group II actions are only affected by corticospinal control to a small extent, whereas the substantial depression of cutaneous FSPs occurs through an effect in the type of cutaneous afferents that are not concerned with presynaptic control. The results indicate that the segmental inputs contributing to group II reflex actions in the lower limb muscles may be relatively free of direct corticospinal control. Corticospinal projection systems are generally considered to command voluntary movement, particularly independent limb movement involving distal limb muscles. The lack of effects here was surprising given that stimulation of corticospinal fibres can produce strong presynaptic effects in other afferents (including cutaneous afferents, as shown in this paper). The absence of direct corticospinal control contrasts with a powerful control of transmission of these afferents through segmental systems and through descending monoaminergic systems (Riddell et al. 1993; Jankowska et al. 1994; Noga et al. 1995). The implications of this are that the control of group II afferent terminals in the midlumbar and sacral segments seems to be controlled in a stereotypical way, presumably associated with specific motor contexts where the actions of the premotor circuitries in those segments are engaged or disengaged.

Acknowledgments Supported by the Wellcome Trust and by NIH grant 5R01NS040863-07.

\section{References}

Aggelopoulos NC, Edgley SA (1995) Segmental localisation of the relays mediating crossed inhibition from group II afferents in the anaesthetized cat spinal cord. Neurosci Lett 185:60-64
Aggelopoulos N, Chakrabarty S, Edgley SA (1999) Transmission from group II afferents in the midlumbar spinal segments is not depressed by stimulation of corticospinal fibres. J Physiol 483:443-460

Andersen P, Eccles JC, Sears TA (1964) Cortically evoked depolarisation of primary afferent fibres in the spinal cord. J Neurophysiol 27:63-77

Arya T, Bajwa S, Edgley SA (1991) Crossed reflex actions from group II muscle afferents in the lumbar spinal cord of the anaesthetised cat. J Physiol 444:117-131

Bras H, Jankowska E, Noga B, Skoog B (1990) Comparison of effects of various types of NA and 5HT agonists on transmission from group II muscle afferents in the cat. Eur J Neurosci 2:1029-1039

Carpenter DA, Lundberg A, Norrsell U (1963) Primary afferent depolarization evoked from the sensorimotor cortex. Acta Physiol Scand 59:126-142

Edgley SA, Jankowska E (1987a) Field potentials generated by group II muscle afferents in the middle lumbar segments of the cat spinal cord. J Physiol 385:393-413

Edgley SA, Jankowska E (1987b) An interneuronal relay for group I and II muscle afferents in the mid-lumbar segments of the cat spinal cord. J Physiol 389:647-674

Edgley SA, Jankowska E, Krutki P, Hammar I (2003) Both dorsal horn and lamina VIII interneurones contribute to crossed reflexes from feline group II muscle afferents. J Physiol 552:961-974

Edgley SA, Jankowska E, Hammar I (2004) Ipsilateral actions of feline corticospinal tract neurons on limb motoneurons. J Neurosci 24:7804-13

Eguibar JR, Quevedo J, Rudomin P (1997) Selective cortical and segmental control of primary afferent depolarization of single muscle afferents in the cat spinal cord. Exp Brain Res 113:411-430

Enríquez M, Jiménez I, Rudomin P (1996) Segmental and supraspinal control of synaptic effectiveness of functionally identified muscle afferents in the cat. Exp Brain Res 107:391-404

Fu T-C, Santini M, Schomburg ED (1974) Characteristics.and distribution of spinal focal synaptic potentials generated by group II muscle afferents. Acta Physiol Scand 91:298-313

Harrison PJ, Jankowska E (1989) Primary afferent depolarization of central terminals of group II muscle afferents in the cat spinal cord. J Physiol 411:71-83

Jack JJ (1978) Some methods for selective activation of muscle afferent fibres. In: Porter R (ed) Studies in neurosphysiology. Cambridge University Press, Cambridge, pp 155-176

Jankowska E, Bichler E, Hammar I (2000) Areas of operation of interneurons mediating presynaptic inhibition in sacral spinal segments. Exp Brain Res 133:402-6

Jankowska E, Lackberg ZS, Dyrehag LE (1994) Effects of monoamines on transmission from group II muscle afferents in sacral segments in the cat. Eur J Neurosci 1:1058-61

Jankowska E, Riddell JS (1993) A relay for input from group II muscle afferents in sacral segments of the cat spinal cord. J Physiol 465:561-580

Jankowska E, Riddell JS (1995) Interneurones mediating presynaptic inhibition of group II muscle afferents in the cat spinal cord. J Physiol 483:461-471

Jankowska E, Riddell JS (1998) Neuronal systems involved in modulating synaptic transmission from group II muscle afferents. In: Rudomin P, Romo R, Mendel L (eds) Presynaptic inhibition and neural control. Oxford University Press, New York, pp 315-328

Jankowska E, Slawinska U, Hammar I (2002a) Differential presynaptic inhibition of actions of group II afferents in di- and polysynaptic pathways to feline motoneurones. J Physiol 542:287-99

Jankowska E, Slawinska U, Hammar I (2002b) On organization of a neuronal network in pathways from group II muscle afferents in feline lumbar spinal segments. J Physiol 542:301-314

Lomeli J, Quevedo J, Linares P, Rudomin P (1998) Local control of information flow in segmental and ascending collaterals of single afferents. Nature 395:600-604 
Lundberg A, Malmgren K, Schomburg ED (1986) Reflex pathways from group II muscle afferents. 1. Distribution and linkage of reflex actions to alpha-motoneurones. Exp Brain Res 65:271-81

Matthews PBC (1972) Mammalian muscle receptors and their central actions. Arnold, London

Noga BR, Jankowska E, Skoog B (1995) Depression of transmission from group II muscle afferents by electrical stimulation of the cuneiform nucleus in the cat. Exp Brain Res 105:25-38

Riddell JS, Hadian M (2000a) Field potentials generated by group II muscle afferents in the lower-lumbar segments of the feline spinal cord. J Physiol 522:97-108

Riddell JS, Hadian M (2000b) Interneurones in pathways from group II muscle afferents in the lower-lumbar segments of the feline spinal cord. J Physiol 522:109-123
Riddell JS, Jankowska E, Eide E (1993) Depolarization of group II muscle afferents by stimuli applied in the locus coeruleus and raphe nuclei of the cat. J Physiol 461:723-741

Riddell JS, Jankowska E, Huber J (1995) Organization of neuronal systems mediating presynaptic inhibition of group II muscle afferents in the cat. J Physiol 483:443-460

Rudomin P, Schmidt RF (1999) Presynaptic inhibition in the vertebrate spinal cord revisited. Exp Brain Res 129:1-37

Rudomin P, Solodkin M, Jiménez I (1986) PAD and PAH response patterns in group Ia- and group Ib-afferent fibers in the cat spinal cord. J Neurophysiol 56:987-1006

Sypert GW, Munson JB, Fleshman JW (1980) Effect of presynaptic inhibition on axonal potentials, terminal potentials, focal synaptic potentials, and EPSPs in cat spinal cord. J Neurophysiol 44:792-803 